\title{
Dome formation on Ceres by solid-state flow analogous to terrestrial salt tectonics
}

\author{
M. T. Bland $\oplus^{1 \star}$, D. L. Buczkowski ${ }^{2}$, H. G. Sizemore ${ }^{3}{ }^{3}$, A. I. Ermakov ${ }^{4}$, S. D. King $\oplus^{5}$, M. M. Sori $\oplus^{6}$, \\ C. A. Raymond $\odot^{4}$, J. C. Castillo-Rogez ${ }^{4}$ and C. T. Russell ${ }^{7}$
}

The dwarf planet Ceres's outer crust is a complex, heterogeneous mixture of ice, clathrates, salts and silicates. Numerous large domes on Ceres's surface indicate a degree of geological activity. These domes have been attributed to cryovolcanism, but that is difficult to reconcile with Ceres's small size and lack of long-lived heat sources. Here we alternatively propose that Ceres's domes form by solid-state flow within the compositionally heterogeneous crust, a mechanism directly analogous to salt tectonics on Earth. We use numerical simulations to illustrate that differential loading of a crust with compositional heterogeneity on a scale of tens of kilometres can produce dome-like features of scale similar to those observed. The mechanism requires the presence of low-viscosity and low-density, possibly ice-rich, material in the upper 1-10 km of the subsurface. Such substantial regional heterogeneity in Ceres's crustal composition is consistent with observations from the National Aeronautics and Space Administration's Dawn mission. We conclude that deformation analogous to that in terrestrial salt tectonics is a viable alternative explanation for the observed surface morphologies, and is consistent with Ceres being both cold and geologically active.

T he surface of the dwarf planet Ceres is dominated by impact craters $^{1}$; however, a range of other geomorphic features are also observed indicating a degree of endogenic geological activity ${ }^{2-4}$. Prominent among these features are $\sim 32$ large domes ${ }^{2,4}$ (Fig. 1) that range from domical with circular planform (for example, Hosil Tholus in Fig. 1a,b) to highly irregular (for example, Liberalia Montes in Fig. 1a). The large domes are typically $20-100 \mathrm{~km}$ in diameter and rise $2-4 \mathrm{~km}$ above the surrounding terrain. Despite their considerable relief, the texture and albedo of the domes' surfaces are generally indistinguishable from the surrounding flatlying, heavily cratered terrain ${ }^{4}$. Although present across a broad latitude range, the domes are primarily clustered in a topographically low region (relative to the best-fit ellipsoid) east of Hanami Planum $\left(270-15^{\circ} \mathrm{E}\right)^{4}$ (Fig. 1). Ahuna Mons also occurs within this region and, although morphologically distinct (taller and steeper) from most of Ceres's large domes ${ }^{5}$ (Supplementary Information), may be the youngest example of such features on Ceres ${ }^{6,7}$. Many domes are not obviously correlated with impact craters; however, several occur within crater interiors (often just interior to the rim), including one of the largest domes, Cosecha Tholus (Fig. 1a,c). Ceres's large domes are one of the clearest indicators of endogenic geological processes on its surface.

Based largely on its high aspect ratio, the 17-km-wide, 4-km-tall Ahuna Mons has been proposed to have formed through cryovolcanic extrusion, analogous to volcanic (dacitic) domes on Earth ${ }^{5}$. This inference was extrapolated to Ceres's other domes ${ }^{6,7}$, leading to a 'cryovolcanism and decay' model in which domes form an initial Ahuna Mons-like edifice by cryovolcanic extrusion and then 'decay' by viscous relaxation into morphologies more typical of Ceres's other domes. Although compellingly argued, sustaining cryovolcanic activity on Ceres is challenging because radiogenic heating is expected to produce a heat flux of just $\sim 1 \mathrm{~mW} \mathrm{~m}^{-2}$ today, and only $\sim 2 \mathrm{~mW} \mathrm{~m}^{-2} 2$ billion years ago ${ }^{8}$. Brines might persist at the base of Ceres's crust but this crust is generally expected to remain rather cold ${ }^{9}$, limiting the prospect for cryovolcanic activity. Furthermore, Ceres's domes lack obvious flow textures or other morphological evidence for an extrusive cryovolcanic origin ${ }^{4}$, and most domes are morphologically quite distinct from Ahuna Mons.

Given these challenges, we propose an alternative model for the formation of Ceres's domes that invokes solid-state flow in a compositionally heterogeneous crust-a mechanism directly analogous to salt tectonics on Earth. Ceres's outer $\sim 40-\mathrm{km}$-thick, low-density crust $^{10}$ is a mixture of hydrated silicates, water ice and a strong lowdensity phase such as clathrates or salts. Globally, water ice composes no more than $\sim 35 \%$ by volume ${ }^{10-12}$. Dawn data further indicate that compositional crustal heterogeneity exists at large scales. Spectroscopic identification of local water ice deposits ${ }^{13}$ suggests exhumation of pockets of ice-rich material that exist in the shallow subsurface ${ }^{14}$, and Dawn gravity data are consistent with crustal density variations over spatial scales of tens to hundreds of kilometres $^{10}$. Impact crater morphology provides evidence for regional variations in crustal density and composition ${ }^{11}$, and the identification of mascons (positive-gravity anomalies associated with mass excesses) beneath some impact craters $^{10}$ may indicate large-scale compositional variations from impact-induced devolatilization ${ }^{15}$. Regional variations in surface composition ${ }^{16}$, and the distribution of features associated with water ice ${ }^{4}$, also imply regional variations in crustal composition.

The resulting picture of Ceres's crust is one in which water ice is not spatially well mixed but is concentrated within localized lenses in the crust. This relatively ice-rich material is lower in viscosity and density than the average crust and is therefore more capable of solid-state flow in response to applied stresses.

\section{The terrestrial salt tectonics analogy}

Our hypothesis for dome formation on Ceres is directly analogous to the formation of salt domes on Earth, in which differential loading drives solid-state flow in low-viscosity, low-density (LVLD) salt. On

IUS Geological Survey, Astrogeology Science Center, Flagstaff, AZ, USA. ${ }^{2} J o h n s$ Hopkins Applied Physics Laboratory, Laurel, MD, USA. ${ }^{3}$ Planetary Science Institute, Tucson, AZ, USA. ${ }^{4}$ Jet Propulsion Laboratory, California Institute of Technology, Pasadena, CA, USA. ${ }^{5}$ Virginia Tech, Blacksburg, VA, USA.

${ }^{6}$ University of Arizona, Tucson, AZ, USA. University of California, Los Angeles, Los Angeles, CA, USA. *e-mail: mbland@usgs.gov 

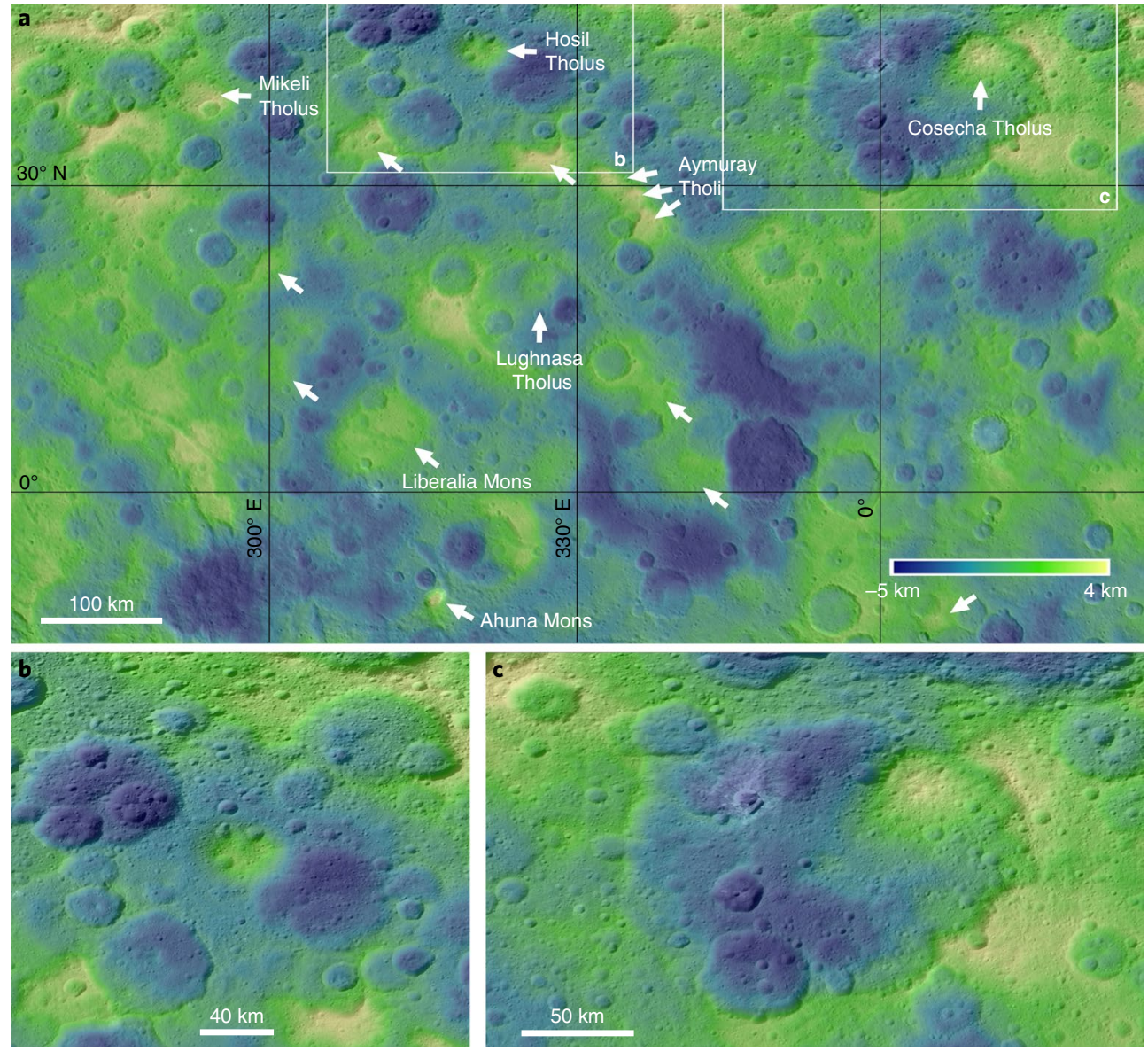

Fig. 1 Ceres's domes (concentrated between $270^{\circ}$ and $15^{\circ} \mathrm{E}$ ) give the surface a distinctive, lumpy appearance. A stereo-derived digital terrain model is overlain on Dawn Framing Camera images ( $140 \mathrm{~m}$ per pixel). a, Domes at regional scale. White arrows indicate the location of prominent domes, some of which are named features (tholi or montes). White boxes indicate the locations of $\mathbf{b}$ (left) and $\mathbf{c}$ (right). $\mathbf{b}$, Close-up of Hosil Tholus (dome in the centre), which occurs in a broad, low region but is otherwise not associated with other surface topography. c, Close-up of Cosecha Tholus, a large dome in the northeast interior of Duginavi crater.

Earth, domes resulting from 'salt tectonics' are a common feature in many regions with substantial buried salt deposits ${ }^{17,18}$. The compositional complexity of Ceres's crust makes it an excellent candidate for application of a salt tectonic-like paradigm. Ceres's location deep in the asteroid belt is unique in that it is cold enough that ice makes up a substantial fraction of the crust (far more than the terrestrial planets), but warm enough that ice can flow over relatively short timescales (in comparison to the icy moons of the giant planets). On Earth, LVLD salts flow relative to higher-viscosity, higher-density (HVHD) sedimentary and crystalline rocks when differential loading occurs. By analogy, on Ceres, LVLD lenses or layers of water icerich material can flow relative to HVHD salts and phyllosilicates.

Terrestrial salt tectonics is primarily driven by differential loading, wherein lateral variations in overburden (gravitational loading) drive underlying salt to flow from areas of high-to-low overburden pressure $^{19}$ (see Supplementary Information). Buoyancy forces are generally recognized to be of secondary importance in initiating diapirs ${ }^{20,21}$. Salt diapirs are thus not primarily formed by RayleighTaylor instabilities, but by fluid pressure. Regional tectonism, such as large-scale extension or contraction of the crust, is critical to the formation of the variety of salt structures observed on Earth. On Ceres, however, regional tectonism appears to be a minor process $^{4,22,23}$ and we therefore focus on differential gravitational loading as a driver for solid-state flow.

Differential loading on Ceres can result from lateral differences in the thickness of a subsurface LVLD layer arising naturally in a poorly mixed crust, or from impact craters that thin the overburden above a pre-existing ice-rich lens. We use the visco-elastic finite element code Tekton2.3 (ref. ${ }^{24}$ ) to simulate the solid-state flow of LVLD material under these two types of differential gravitational loading (see Methods). For simplicity, we assume that the LVLD material has the properties of water ice and that the HVHD material has the properties of Ceres's average crust. The effect of viscosity and density on our model results is discussed in the Supplementary Information. In case 1, we posit a LVLD layer at depth with strong lateral variation in thickness (Fig. 2). Differential loading is created by the lateral difference in overburden thickness. These simulations are directly analogous to early models of salt dome formation on Earth $^{25}$. In case 2, we posit a uniformly thick LVLD lens of material buried beneath a portion of an impact crater (Fig. 4). Ice is assumed to have been removed from directly beneath the crater by impact heating ${ }^{15}$. Here the difference in overburden thickness due to crater topography creates a hydraulic head that drives flow. In both cases, the mass anomalies associated with the presence of the LVLD material are sufficiently small to be unresolvable in current gravity data (Supplementary Information).

\section{Dome formation driven by differential loading}

In both types of simulation, subsurface solid-state flow produces domes similar in scale to those observed on Ceres. Figure 2 shows our reference case 1 simulation: differential loading due to lateral variations in the thickness of a LVLD layer at depth. In this case, 

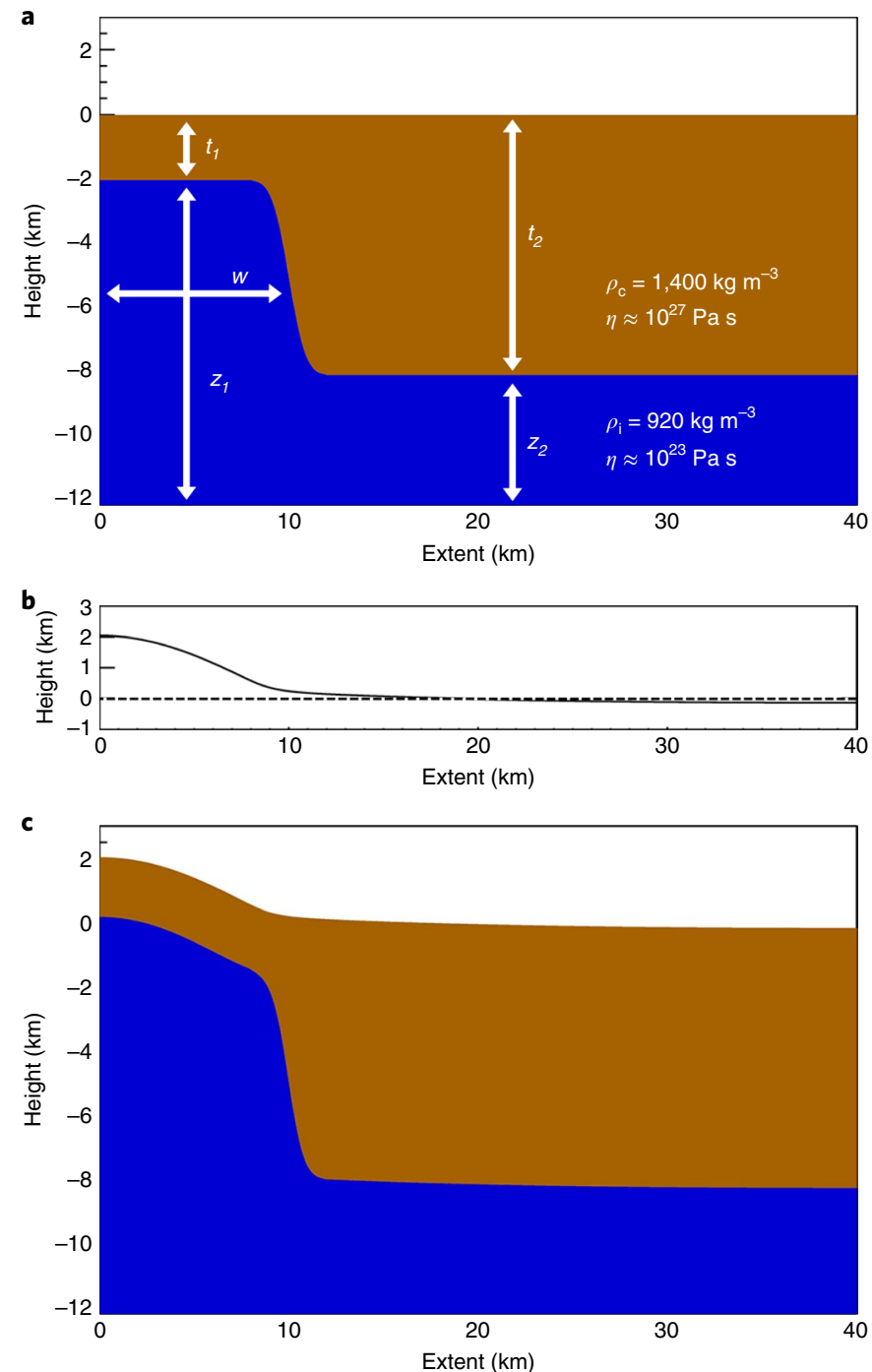

Fig. 2 | Reference simulation evaluating the effectiveness of differential gravitational loading due to lateral variations in the thickness of a subsurface layer of LVLD material (case 1). a, The initial geometry, here shown in cross-section, consists of a LVLD layer (blue) buried in the crust (brown). The layer is $4 \mathrm{~km}$ thick and the top is at $8 \mathrm{~km}$ depth. A $20-\mathrm{km}$ wide ( $w$, axisymmetric), 6-km-tall column $\left(z_{1}-z_{2}\right)$ extends to within $2 \mathrm{~km}$ of the surface. The surface is initially flat (dashed line in $\mathbf{b}$ ). The difference in crustal thickness $\left(\Delta t=t_{2}-t_{1}\right)$ is $6 \mathrm{~km}$. b,c, The resulting surface $(\mathbf{b})$ and subsurface (c) deformation after $10 \mathrm{Myr}$ of differential loading. Upward and lateral flow has resulted in a dome $2 \mathrm{~km}$ tall. Vertical exaggeration is $\sim 1.75 \mathrm{X}$.

deformation is driven by the 6-km difference in overburden thickness, which creates a strong gradient in hydraulic head. Figure $2 b, c$ indicates that a dome $20 \mathrm{~km}$ across rises to a height of just over $2 \mathrm{~km}$ in 10 million years (Myr). Although dome growth is not complete in the simulation shown (Fig. 3), total dome height is limited by several factors including the removal of the hydraulic gradient (that is, dome height is self-limited even in the absence of other resistance), the flexural rigidity of the overlying crust, which resists bending, and drag along the LVLD/HVHD boundary (Supplementary Information).

To better understand the range of dome-like features that can be produced by differential loading of this sort, we performed a broad suite of simulations designed to investigate how the geometry of the LVLD material affects dome formation (Supplementary
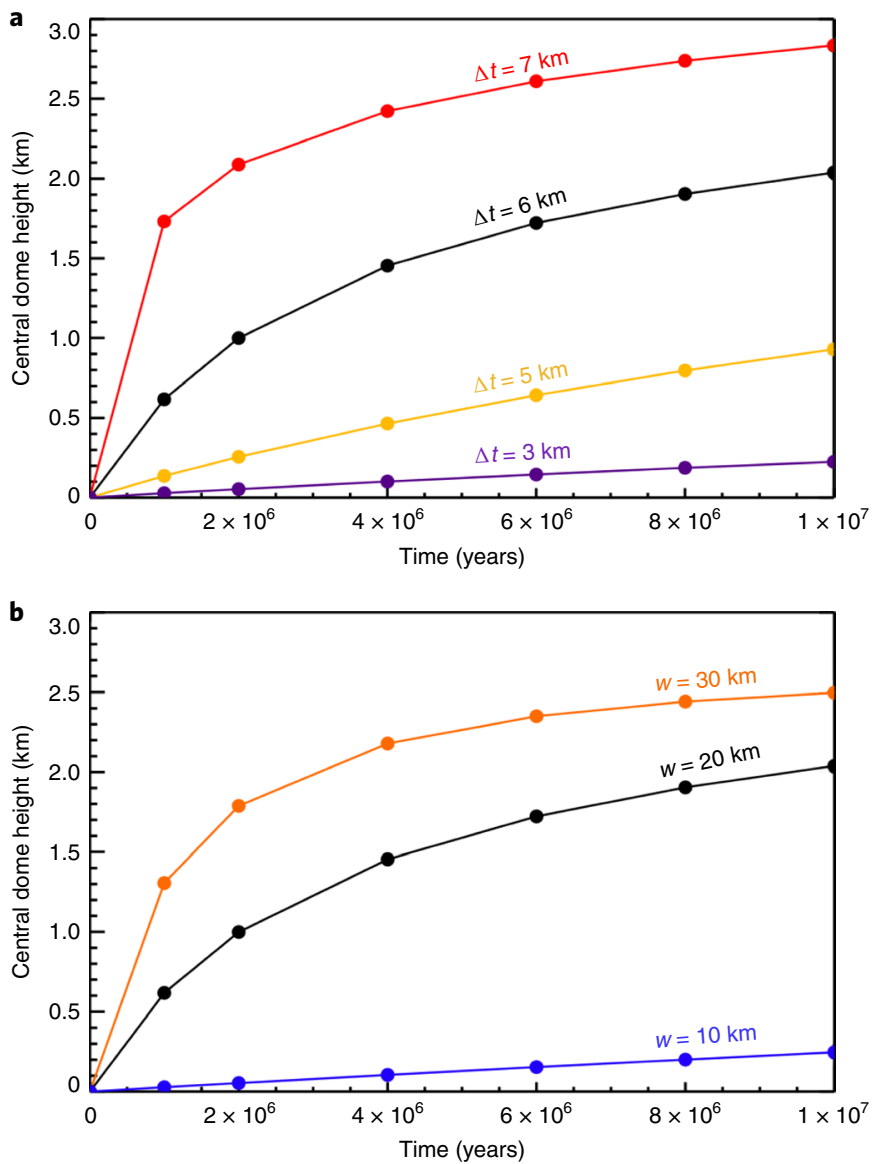

Fig. 3 | Dome amplitude as a function of time for several case 1 simulations. a, Demonstration of the effect of overburden thickness on dome growth. The black curve is the reference case 1 simulation (Fig. 2); the red, yellow and purple curves denote $\Delta t$ as indicated. $\mathbf{b}$, Demonstration of the effect of subsurface column width $(w)$ on dome growth.

Information). Geometries that maximize the lateral difference in overburden thickness $(\Delta t)$ have greater differential loading and result in taller domes (Fig. 3a). Thus, the ideal geometry includes a deeply buried layer and a column that approaches the surface. For example, a simulation with $\Delta t=7 \mathrm{~km}$ results in a dome that rises to a height of nearly $3 \mathrm{~km}$ (red curve in Fig. 3a). In contrast, geometries that minimize $\Delta t$ result in less growth. For example, a simulation with $\Delta t=5 \mathrm{~km}$ results in a dome only $930 \mathrm{~m}$ tall (yellow curve in Fig. 3a). Column width $(w)$ also plays a critical role in the amplitude of the resulting domes (Fig. 3b). Wider subsurface columns of LVLD material result in larger amplitude domes than narrower columns. For example, geometries identical to the reference simulation but with widths of 30 and $10 \mathrm{~km}$ result in domes that are $2.5 \mathrm{~km}$ and $200 \mathrm{~m}$ tall, respectively (Fig. 3b). The growth of domes from narrow columns of subsurface material is limited, in part, by the flexural rigidity of the lithosphere, which retards dome formation when column width is $20-30 \mathrm{~km}$, and prevents it entirely when the width is $<10 \mathrm{~km}$ (Supplementary Information).

Subsurface solid-state flow also readily occurs when the source of differential loading is surface topography. Figure 4 shows the results of our case 2 reference simulation. In $<1$ Myr a dome $3.6 \mathrm{~km}$ high has formed in the bottom of the crater. The dome is directly analogous in height and location to Cosecha Tholus within Duginavi crater (Fig. 4e), and larger than Lughnasa Tholus in Begbalel crater (Fig. 1a). As in the simulations of isolated domes (Fig. 2), we varied the geometry of the LVLD layer to evaluate how the thickness 

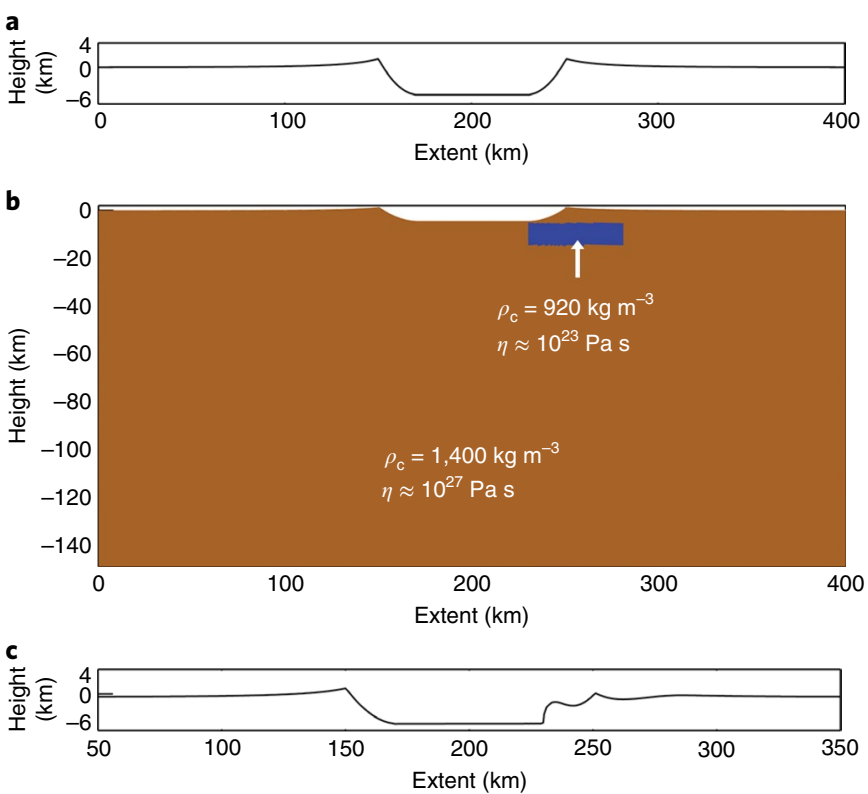

d

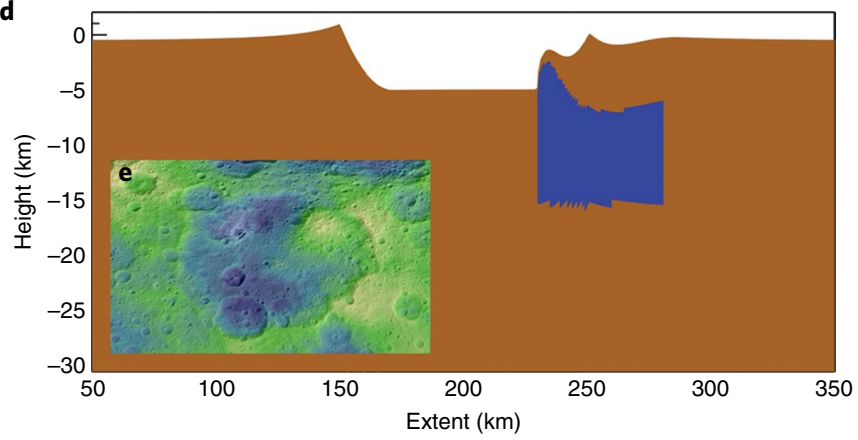

Fig. 4 | Reference simulation evaluating the effectiveness of surface topography in generating differential gravitational loading of a uniform buried layer (case $\mathbf{2}$ ). $\mathbf{a}, \mathbf{b}$, The initial geometry, here shown in crosssection, consists of a 10-km-thick LVLD layer positioned $5 \mathrm{~km}$ beneath the surface, and just $500 \mathrm{~m}$ below the floor of a $4.5-\mathrm{km}$-deep, $100-\mathrm{km}-$ diameter impact crater. $\mathbf{c}, \mathbf{d}$, The final result of the simulation in which loading results in a $3.6-\mathrm{km}$-high dome within the crater interior. $\mathbf{e}, \mathrm{An}$ analogous structure on Ceres: Cosecha Tholus within Duginavi crater.

of the layer, its depth beneath the surface and crater floor, and its lateral position relative to the crater centre, affected dome formation (Supplementary Information). We find that dome formation is inhibited for either a deeper or thinner layer. For deeper layers, the flexural rigidity of the thick overburden is increased, increasing resistance to flow. Increasing the layer depth to $10 \mathrm{~km}$ (that is, twice as deep as our nominal case) produces negligible doming in the crater floor. Likewise, decreasing the layer thickness to $5 \mathrm{~km}$ (that is, half the layer thickness of our nominal case) produces only a small dome, $1.4 \mathrm{~km}$ tall relative to the crater floor. Interestingly, a layer that extends beneath the entire crater can produce vertical uplift of the crater floor, with small domes forming at the base of the crater walls (Supplementary Information). This type of uplift might be associated with the floor-fracturing observed in some large craters on Ceres $^{23}$. Shallower craters have smaller hydraulic gradients due to smaller differences in overburden thickness, and therefore result in smaller domes. Deeper craters, in contrast, result in larger domes.

Implications for cryovolcanism and Ceres's geomorphology The mechanism we propose for the formation of Ceres's domes provides an alternative to a cryovolcanic origin. The cryovolcanic hypothesis is consistent with the observed shapes of the domes and the fact that the youngest dome (Ahuna Mons) is also the steepest ${ }^{6,7}$, but requires geologically recent igneous activity. The attractiveness of our salt tectonic-like mechanism is that it does not require Ceres's crust to remain warm over geological time ${ }^{7}$. Instead, Ceres's domes are a natural result of compositional spatial heterogeneity in the upper crust. In this paradigm, Ceres could have been 'frozen' (or nearly $\mathrm{so}^{12}$ ) billions of years ago yet remain geologically active today.

Salt tectonic-like deformation on Ceres requires that LVLD material occurs locally within the upper few kilometres of the crust in regions where large domes occur. Dawn data, including the spectral detection of local deposits of water ice ${ }^{13}$, gravity data ${ }^{10}$, crater morphology $\mathrm{y}^{11}$ and the existence of the domes themselves ${ }^{4}$, indicate that localized regions of more ice-rich material exist in the subsurface; however, its origin (along with the origin of the crust in general $^{26}$ ) remains an open question. On Earth, evaporite (salt) layers are typically deposited as thick sequences over millions of years and then subsequently buried by sediments ${ }^{27}$. No such mechanism is interpreted to have acted on Ceres. Instead, buoyancy-driven flow through fractures or macropore space over tens or hundreds of millions of years may have permitted low-density material to accumulate at depths of a few to $\sim 10 \mathrm{~km}$. In that case, regions of initially higher porosity might have accumulated greater ice content. Similarly, brines like those invoked for cryovolcanism ${ }^{5,928,29}$ may have infiltrated portions of the porous crust and refroze to form broad regions of relatively low-density material in the subsurface.

The concentration of domes in Ceres's topographically low regions may provide a clue to their origin. These low regions may be of impact origin ${ }^{30}$. Such a global-scale impact would have exhumed deeper material ${ }^{31}$, potentially including ice-rich LVLD material remaining from Ceres's incomplete differentiation ${ }^{32}$. The postimpact crustal structure might then include LVLD material in the shallow subsurface. Alternatively, the impact could have destabilized clathrates in the crust, converting a relatively strong crustal component to much weaker water ice ${ }^{26}$. Whether of impact origin or not, the topographic lows are also likely regions of thinner crust ${ }^{10}$, which again suggests that domes form when an originally deeper, perhaps more heterogeneous, layer of the crust is brought nearer the surface.

Similarly, smaller impacts might also have enabled dome formation by further un-roofing LVLD material. Such material would have been removed directly beneath the crater by impact heating and crater excavation flow ${ }^{33}$; however, LVLD material might have persisted beneath the final crater rim (Fig. 4), outside the high temperatures and pressures of the transient impact cavity. Postimpact warming might also have sufficiently decreased viscosities to enable increased flow for millions of years ${ }^{33}$. Such a scenario provides a simple explanation for the domes in Duginavi crater (Cosecha Tholus), Begbalal crater (Lughnasa Tholus) and other local topographic lows (for example, south of Azacca crater).

It remains uncertain whether Ahuna Mons, Ceres's most iconic edifice, and Yamor Mons, a similarly shaped dome, could have been formed by a salt tectonics-like mechanism. In principle, solid-state flow can reproduce Ahuna and Yamor Mons' 3-4 km of relief and high aspect ratios given the right subsurface geometry and sufficient density variations (Supplementary Information). The requirements (a very thin overburden and deep layer) are extreme, but may indicate the rarity of such structures on Ceres. A related possibility is that Ahuna Mons and Yamor Mons represent a mode of solid-state doming referred to as piercement, during which a thin overburden is fractured, forcibly lifted and rotated aside ${ }^{19,34,35}$. LVLD material can then breach the surface, driven by subsurface fluid pressure. In terrestrial settings this breached material spreads laterally, limiting dome height; however, the colder surface and compositionally unique material on Ceres might have resulted in a different morphology. This mode of diapirism is consistent with the observation of upturned deposits of pre-existing regolith on the flank of Ahuna 
Mons ${ }^{5}$ and its distinctive, sodium carbonate-rich (relative to the average crust) composition ${ }^{36}$.

Our hypothesis for dome formation does not prohibit the occurrence of cryovolcanism on Ceres, and we cannot unequivocally eliminate the possibility that Ahuna Mons, with its relatively distinct morphology relative to Ceres's other domes, formed by cryovolcanic activity. Like Earth, Mars and the icy moons, Ceres's is complex. A single mechanism may not explain every large dome on the dwarf planet. Ultimately, both cryovolcanism and ice tectonics may have occurred on Ceres, with different domes having formed by one process or the other.

\section{Online content}

Any methods, additional references, Nature Research reporting summaries, source data, statements of code and data availability and associated accession codes are available at https://doi.org/10.1038/ s41561-019-0453-0.

Received: 21 December 2018; Accepted: 20 August 2019; Published online: 27 September 2019

\section{References}

1. Hiesinger, H. et al. Cratering on Ceres: implications for its crust and evolution. Science 353, aaf4759 (2016).

2. Buczkowski, D. L. et al. The geomorphology of Ceres. Science 353, aaf4332 (2016)

3. Schmidt, B. E. et al. Geomorphological evidence for ground ice on dwarf planet Ceres. Nat. Geo. 10, 338-343 (2017).

4. Sizemore, H. G. et al. A global inventory of ice-related morphological features on dwarf planet Ceres: implications for the evolution and current state of the cryosphere. J. Geophys. Res. 124, 1650-1689 (2019).

5. Ruesch, O. et al. Cryovolcanism on Ceres. Science 353, aaf4286 (2016).

6. Sori, M. M. et al. The vanishing cryovolcanoes of Ceres. Geophys. Res. Lett. 44, 1243-1250 (2017).

7. Sori, M. M. et al. Cryovolcanic rates on Ceres revealed by topography. Nat. Astro. 2, 946-950 (2018)

8. Bland, M. T. Predicted crater morphologies on Ceres: probing internal structure and evolution. Icarus 226, 510-521 (2013).

9. Castillo-Rogez, J. C. et al. Conditions for the long-term preservation of a deep brine reservoir in Ceres. Geophys. Res. Lett. 46, 1963-1972 (2018)

10. Ermakov, A. I. et al. Constraints on Ceres' internal structure and evolution from its shape and gravity measured by the Dawn spacecraft. J. Geophys. Res. 122, 1-27 (2017).

11. Bland, M. T. et al. Composition and structure of the shallow subsurface of Ceres revealed by crater morphology. Nat. Geo. 9, 538-542 (2016).

12. Fu, R. R. et al. The interior structure of Ceres as revealed by surface topography. Earth Planet. Sci. Lett. 476, 153-164 (2017).

13. Combe, J.-P. et al. Detection of local $\mathrm{H}_{2} \mathrm{O}$ exposed at the surface of Ceres. Science 353, aaf3010 (2016).

14. Hughson, K. H. G. et al. The Ac-5 (Fejokoo) quadrangle of Ceres: geologic map and geomorphological evidence for ground ice mediated surface processes. Icarus 316, 63-83 (2018).

15. Bland, M. T. et al. Morphological indicators of a mascon beneath Ceres's largest crater, kerwan. Geophys. Res. Lett. 45, 1297-1304 (2018).

16. Ammannito, E. et al. Distribution of phyllosilicates on the surface of Ceres. Science 353, aaf4279 (2016).

17. Peel, F. J., Travis, C. J. \& Hossack, J. R. in Salt Tectonics: A Global Perspective (eds Jackson, M. P. A. et al.) 153-175 (AAPG, 1995).

18. Jackson, M. P. A. et al. Salt Diapirs of the Great Kavir, Central Iran (Geological Society of America, 1990).

19. Hudec, M. R. \& Jackson, M. P. A. Terra infirma: understanding salt tectonics. Earth Sci. Rev. 82, 1-28 (2007)

20. Jackson, M. P. A. in Salt Tectonics: A Global Perspective (eds Jackson, M. P. A. et al.) 1-28 (AAPG, 1995).
21. Jackson, M. P. A. Conceptual Breakthroughs in Salt Tectonics: A Historical Review, 1856-1993 (Univ. Texas at Austin, Bureau of Economic Geology, 1997).

22. Scully, J. E. C. et al. Evidence for the interior evolution of Ceres from geologic analysis of fractures. Geophys. Res. Lett. 44, 9564-9572 (2017).

23. Buczkowski, D. L. et al. Floor-fractured craters on Ceres and implications for interior processes. J. Geophys. Res. 123, 3188-3204 (2018).

24. Melosh, H. J. \& Raefsky, A. The dynamical origin of subduction zone topography. Geophys. J. Int. 60, 333-334 (1980).

25. Schultz-Ela, D. D., Jackson, M. P. A. \& Vendeville, B. C. Mechanics of active salt diapirism. Tectonophysics 228, 275-312 (1993).

26. Castillo-Rogez, J. C. et al. Insights into Ceres's evolution from surface composition. Meteorit. Planet. Sci. 53, 1820-1843 (2018).

27. Schreiber, B. C. \& El Tabakh, M. Deposition and early alteration of evaporites. Sedimentology 47, 215-238 (2000).

28. Quick, L. et al. A possible brine reservoir beneath occator crater: thermal and compositional evolution and formation of the Cerealia dome and Vinalia faculae. Icarus 320, 119-135 (2018).

29. Ruesch, O. et al. Bright carbonate surfaces on Ceres as remnants of salt-rich water fountains. Icarus 320, 39-48 (2019).

30. Marchi, S. et al. The missing large impact craters on Ceres. Nat. Commun. 7 , 12257 (2016).

31. Jutzi, M., Asphaug, E., Gillet, P., Barrat, J.-A. \& Benz, W. The structure of the asteroid 4 Vesta as reveled by models of planet-scale collisions. Nature 494, 207-210 (2013)

32. Park, R. S. et al. A partially differentiated interior for (1) Ceres deduced from its gravity field and shape. Nature 537, 515-517 (2016).

33. Bowling, T. J. et al. Post-impact thermal structure and cooling timescale of Occator crater on asteroid 1 Ceres. Icarus 320, 110-118 (2019).

34. Vendeville, B. C. \& Jackson, M. P. A. The rise of diapirs during thin-skinned extension. Mar. Pet. Geol. 9, 331-354 (1992).

35. Weijermars, R., Jackson, M. P. A. \& Vendeville, B. Rheological and tectonic modeling of salt diapirs. Tectonophysics 217, 143-174 (1993).

36. Zambon, F. et al. Spectral analysis of Ahuna Mons from Dawn mission's visible-infrared spectrometer. Geophys. Res. Lett. 44, 97-104 (2016).

\section{Acknowledgements}

This work was supported by the National Aeronautics and Space Administration's (NASA's) Dawn Guest Investigator Program (grant no. NNH15AZ85I). Some of the research was carried out at the Jet Propulsion Laboratory, California Institute of Technology, under a contract with NASA. Special thanks to the Dawn mission operations team, who have gone above and beyond to return exceptional data from Ceres. Any use of trade, firm or product names is for descriptive purposes only and does not imply endorsement by the US Government.

\section{Author contributions}

M.T.B. performed the numerical simulations and drafted the manuscript. D.L.B. and H.G.S provided detailed comments on the text and morphological information for Ceres's large domes. A.I.E. and S.D.K. provided modelling of the gravity anomaly associated with subsurface density variations. M.M.S. and J.C.C.-R. provided insight into cryovolcanism on Ceres and comments on the text. C.A.R. provided insight into Ceres crustal heterogeneity. C.T.R. and C.A.R. are responsible for the success of the Dawn mission and provided data access.

\section{Competing interests}

The authors declare no competing interests.

\section{Additional information}

Supplementary information is available for this paper at https://doi.org/10.1038/ s41561-019-0453-0.

Correspondence and requests for materials should be addressed to M.T.B.

Peer review information Primary Handling Editor(s): S. Lachowycz

Reprints and permissions information is available at www.nature.com/reprints.

Publisher's note Springer Nature remains neutral with regard to jurisdictional claims in published maps and institutional affiliations.

This is a U.S. government work and not under copyright protection in the U.S.; foreign copyright protection may apply 2019 


\section{Methods}

We used the finite element code Tekton2.3 (ref. ${ }^{24}$ ) to simulate the solid-state flow of LVLD material under differential loading in two dimensions. For simplicity we simulated two distinct layers, whereas in reality compositional gradients and mixing can occur. These complications are discussed in the Supplementary Information. As described above, we simulated two broad cases in which differential loading might have occurred on Ceres. The first posits a LVLD layer at depth with a lateral variation in thickness (Fig. 2). The simulations were performed in an axisymmetric geometry in which the sides of the domain were fixed in the horizontal and free in the vertical, and the bottom of the domain was fixed in both the horizontal and vertical. A horizontal free-slip boundary at the bottom of the domain did not change our qualitative results. The top of the domain was a free surface. The domain was a total of $12 \mathrm{~km}$ in depth and $40 \mathrm{~km}$ in length, and the finite element mesh had a horizontal resolution of $100 \mathrm{~m}$ and a vertical resolution that varied between 40 and $160 \mathrm{~m}$. A total of 48,000 elements were used. The simulations are directly analogous to early models of salt dome formation on Earth ${ }^{25}$. The second type of simulation posits a uniformly thick LVLD layer buried beneath topography consistent with an impact crater (Fig. 4). The boundary conditions were identical to those in the first case, but the simulations were performed in plane-strain due to their asymmetric geometry. Very similar results were obtained if an axisymmetric geometry was assumed with an annulus of LVLD material in the subsurface; however, the resulting deformation produced a toroidal uplift at the base of the crater walls (due to the axisymmetry), which is not observed on Ceres. In this case, the domain was $150 \mathrm{~km}$ deep and $400 \mathrm{~km}$ long, necessitated by the size of the crater, with a horizontal resolution of $1 \mathrm{~km}$ and a vertical resolution that varied from $75 \mathrm{~m}$ to $4 \mathrm{~km}$. A total of 40,000 elements were used in these simulations. In both types of simulation, the domain was allowed to deform under a gravitational acceleration of $0.27 \mathrm{~m} \mathrm{~s}^{-2}$ for up to $10 \mathrm{Myr}$ (long enough to evaluate dome growth with reasonable computation time). Longer timescales were investigated for the lower-viscosity simulations described in the Supplementary Information.

The model is visco-elastic and, for simplicity we assumed the LVLD has the properties of water ice $\left(\rho_{\mathrm{i}}=920 \mathrm{~kg} \mathrm{~m}^{-3}\right)$ and the HVHD material has the properties of Ceres's average crust $\left(\rho_{\mathrm{c}}=1.400 \mathrm{~kg} \mathrm{~m}^{-3}\right)$. The crustal density is modestly higher than the average density ${ }^{10}\left(1,287^{-87}+70\right)$ but is consistent with a crust locally depleted in volatiles (for example, accounting for a segregated low-density component). Only the ratio of the densities, rather than their absolute values, is important (Supplementary Information) - the same result is obtained if both densities are increased by the same amount (for example, to permit a LVLD composition other than pure water ice). A lower density ratio limits the hydraulic gradient and the resulting dome height (Supplementary Information). Both layers used identical elastic parameters-Young's modulus of $9.33 \mathrm{GPa}$ and a Poisson's ratio of 0.325 . The simulations assumed an ice-like rheology and included all appropriate flow mechanisms for ice I (ref. ${ }^{37}$ ), including dislocation creep (three regimes), grainboundary-sliding, basal slip and diffusion creep. Each flow mechanism is described by a non-Newtonian Arrhenius law, summed such that each flow mechanism acts simultaneously (grain-boundary sliding and basal slip are rate limiting and so add in parallel; see ref. ${ }^{37}$ for parameter values). We assumed a grain size of $1 \mathrm{~mm}$. Because the rheology is stress dependent, a single viscosity cannot be obtained for comparison to, for example, ref. ${ }^{12}$. A posteriori analysis indicates that the effective viscosity of the crust was $\sim 10^{27}-10^{28} \mathrm{~Pa}$ s, modestly higher than the $10^{25} \mathrm{Pas}$ determined in ref. ${ }^{12}$. However, our results are not strongly dependent on the viscosity of the crust, and a lower viscosity would promote only more rapid dome formation (although not taller domes). The effective viscosity of the LVLD layer was $10^{22}-10^{23} \mathrm{Pas}$, consistent with clean ice at $145-150 \mathrm{~K}$, or warmer but dirty ice. The effect of viscosity, which can also be affected by the inclusion of particulates (rock or salt) ${ }^{38,39}$, is discussed further in the Supplementary Information.

A sensitivity analysis indicates that the inclusion of plasticity (brittle behaviour) does not modify our basic results. In all cases, we did not include thermodynamics and neglected any effect of temperature variations with depth on density or viscosity. Ceres's low radiogenic heat flux and the shallow depths with which we are concerned here warrant such an approximation. A temperature was chosen for the crust and LVLD, respectively, such that typical stresses resulted in the effective viscosities above.

\section{Data availability}

All data used in the study are available in NASA's Planetary Data System archive (Small Bodies Node: https://pds-smallbodies.astro.umd.edu/).

\section{Code availability}

The original Tekton 2.3 source code used in the simulations presented is no longer publicly available. Please contact the corresponding author for additional information.

\section{References}

37. Durham, W. B. \& Stern, L. A. Rheological properties of water ice: applications to satellites of the outer planets. Annu. Rev. Earth Planet. Sci. 28 , 295-330 (2001).

38. Durham, W. B., Pathare, A. V., Stern, L. A. \& Lenferink, H. J. Mobility of icy sand packs, with application to Martian permafrost. Geophys. Res. Lett. 36, L23203 (2009).

39. Mangold, N., Allemand, P., Duval, P., Geraud, Y. \& Thomas, P. Experimental and theoretical deformation of ice-rock mixtures: implications on rheology and ice content of Martian permafrost. Planet. Space Sci. 50, 385-401 (2002). 